\title{
Reversible Markov chains and optimality of symmetrized empirical estimators
}

\author{
PRISCILLA E. GREENWOOD ${ }^{1}$ and WOLFGANG WEFELMEYER ${ }^{2}$ \\ ${ }^{1}$ Department of Mathematics, University of British Columbia, 121-1984 Mathematics Road, \\ Vancouver, B.C., Canada V6T 1Z2.e-mail: pgreenw@math.ubc.ca \\ ${ }^{2}$ Universität - Gesamthochschule Siegen, Fachbereich 6 Mathematik, Walter-Flex-Str. 3, 57068 \\ Siegen, Germany.e-mail:wefelmeyer@mathematik.uni-siegen.de
}

\begin{abstract}
Suppose that we want to estimate the expectation of a function of two arguments under the stationary distribution of two successive observations of a reversible Markov chain. Then the usual empirical estimator can be improved by symmetrizing. We show that the symmetrized estimator is efficient. We point out applications to discretely observed continuous-time processes. The proof is based on a result for general Markov chain models which can be used to characterize efficient estimators in any model defined by restrictions on the stationary distribution of a single or two successive observations.
\end{abstract}

Keywords: discretely observed diffusions; efficient estimation; inference for stochastic processes; martingale approximation

\section{Introduction}

Suppose that we observe $X_{0}, \ldots, X_{n}$ from an ergodic Markov chain with unknown transition distribution $Q(x, \mathrm{~d} y)$ and invariant distribution $\pi(\mathrm{d} x)$. We want to estimate the expectation of a function $f(x, y)$ under the joint stationary distribution $(\pi \otimes Q)(\mathrm{d} x, \mathrm{~d} y)=\pi(\mathrm{d} x) Q(x, \mathrm{~d} y)$ of two successive observations. Greenwood and Wefelmeyer (1995) show that the empirical estimator

$$
E_{n} f=\frac{1}{n} \sum_{i=1}^{n} f\left(X_{i-1}, X_{i}\right)
$$

is asymptotically efficient.

Suppose now that we know the chain to be reversible:

$$
\pi(\mathrm{d} x) Q(x, \mathrm{~d} y)=\pi(\mathrm{d} y) Q(y, \mathrm{~d} x) .
$$

This means that the joint stationary distribution of two successive observations is symmetric in the two components. Hence the empirical estimator can be improved by symmetrizing. The symmetrized empirical estimator is

$$
E_{n}^{s} f=\frac{1}{2 n} \sum_{i=1}^{n}\left\{f\left(X_{i-1}, X_{i}\right)+f\left(X_{i}, X_{i-1}\right)\right\} .
$$

We show that this estimator is asymptotically efficient. 
For a function $f(x)$ of one argument, the symmetrized empirical estimator is asymptotically equivalent to the ordinary estimator $(1 / n) \sum_{i=1}^{n} f\left(X_{i}\right)$. Hence our result implies that reversibility carries no information on the expectation of such a function.

The proof is based on a characterization of efficient estimators in any model defined by a restriction on the stationary distribution of a single or two successive observations. Existing efficiency results for ergodic Markov chains have been for two types of model: fully nonparametric models, and models in which the transition distribution is parametrized. Penev (1991) and Bickel (1993) consider estimating $\pi f$ and Greenwood and Wefelmeyer (1995) consider estimating $\pi \otimes Q f$ for models of the first type, when nothing is known about $Q$. The main examples of the second type are autoregressive processes, which can be described by parametric or semiparametric models for their transition distributions. For efficient estimation see, for example, Kreiss (1987), Jeganathan (1995), Koul and Schick (1997) and Drost and Klaassen (1996). Autoregressive processes with non-independent innovations have been treated by Wefelmeyer (1996). The techniques for the type of model considered here, where the restriction is on the stationary law, are different.

An application of our result is to discretely observed diffusions. Suppose that we have a nonparametric model for a diffusion process:

$$
\mathrm{d} X_{t}=a\left(X_{t}\right) \mathrm{d} t+\sigma\left(X_{t}\right) \mathrm{d} W_{t} .
$$

If we observed the process at evenly spaced discrete time points, then the observations form a homogeneous Markov chain. The transition distribution is usually difficult to calculate, and we may be interested in finding the best estimator that does not require this calculation. If $a$ and/or $\sigma$ vary freely, the corresponding class of invariant distributions includes all distributions with (smooth) positive densities. Since such diffusion processes are reversible, so are the corresponding discretely observed diffusions, and our model consists of reversible Markov chains. A recent review of continuous-time Markov processes has been given by Hansen and Scheinkman (1995). Estimators for parametric models of discretely observed diffusions have been studied by Bibby and Sørensen (1995) and Pedersen (1995), and for nonparametric models of discretely observed diffusions by Aït-Sahalia (1996).

In Section 2 we recall a characterization of efficient estimators for the full nonparametric Markov chain model and arbitrary submodels. In Section 3, Theorem 1, we specialize the characterization to submodels defined by restrictions on the stationary distribution of a single or two successive observations. We also describe briefly an application to models defined by restricting the stationary distribution of a single observation to a parametric family. Such models arise when a diffusion is discretely observed and the diffusion model is parametric. Details will be given in a forthcoming paper by Kessler, Schick and Wefelmeyer (1997). In Section 4, Theorem 2, we prove the efficiency of the symmetrized empirical estimator by applying Theorem 1 to the submodel given by all reversible Markov chains. Section 5 contains the proofs of the lemmas.

\section{Full model and submodel}

In this section we recall a characterization of efficient estimators for arbitrary smooth functionals of the transition distribution in general Markov chain models. The characteriza- 
tion is based on a nonparametric version of Hájek's (1970) convolution theorem. It requires the model to be locally asymptotically normal.

We begin with the full nonparametric model, in which no restrictions are made on the transition distribution. Let $X_{0}, \ldots, X_{n}$ be observations from a Markov chain with values in some measurable space. We shall introduce a local parameter space at a fixed transition distribution $Q(x, \mathrm{~d} y)$ such that the chain satisfies the two assumptions below.

Assumption 1. The chain is positive Harris recurrent.

Let $\pi(\mathrm{d} x)$ denote the invariant distribution. For a suitably integrable function $f_{1}(x)$ of one argument write

$$
\left(Q f_{1}\right)(x)=\int Q(x, \mathrm{~d} y) f_{1}(y), \quad \pi f_{1}=\int \pi(\mathrm{d} x) f_{1}(x) .
$$

For a function $f(x, y)$ of two arguments we shall write

$$
(Q f)(x)=\int Q(x, \mathrm{~d} y) f(x, y), \quad Q^{j} f=Q^{j-1} Q f, \quad \pi \otimes Q f=\iint \pi(\mathrm{d} x) Q(x, \mathrm{~d} y) f(x, y) .
$$

Assumption 2. The chain has the ergodicity property

$$
\sup \left\{\pi\left(Q^{j} f_{1}-\pi f_{1}\right)^{2}: \pi f_{1}^{2} \leqslant 1\right\} \rightarrow 0 \quad \text { for } j \rightarrow \infty .
$$

The space

$$
H=\left\{h \in L_{2}(\pi \otimes Q): Q h=0\right\}
$$

is a local parameter space in the sense that for each $h \in H$ we can choose a sequence $Q_{n h}$ which is Hellinger differentiable with derivative $h$ :

$$
\int Q(x, \mathrm{~d} y)\left(\frac{\mathrm{d} Q_{n h}}{\mathrm{~d} Q}(x, y)^{1 / 2}-1-n^{-1 / 2} \frac{1}{2} h(x, y)\right)^{2} \leqslant n^{-1} r_{n}(x)
$$

with $r_{n}$ decreasing to 0 pointwise and $\pi$ integrable for large $n$. This version of differentiability is due to Höpfner et al. (1990).

Remark. In the full model we can take

$$
Q_{n h}(x, \mathrm{~d} y)=Q(x, \mathrm{~d} y)\left\{1+n^{-1 / 2} h_{n}(x, y)\right\},
$$

with

$$
h_{n}=\bar{h}_{n}-Q \bar{h}_{n} \quad \text { and } \quad \bar{h}_{n}=h 1_{\left(|h| \leqslant n^{1 / 8}\right)} \text {. }
$$

Write $P_{n}$ and $P_{n h}$ for the joint distribution of $X_{0}, \ldots, X_{n}$ under $Q$ and $Q_{n h}$, respectively. As in Höpfner (1993) we have a nonparametric version of local asymptotic normality: 


$$
\log \left(\frac{\mathrm{d} P_{n h}}{\mathrm{~d} P_{n}}\right)=n^{-1 / 2} \sum_{i=1}^{n} h\left(X_{i-1}, X_{i}\right)-\frac{1}{2} \pi \otimes Q h^{2}+o_{P_{n}}(1)
$$

and

$$
n^{-1 / 2} \sum_{i=1}^{n} h\left(X_{i-1}, X_{i}\right) \Rightarrow N_{h}
$$

where $N_{h}$ is normal with mean 0 and variance $\pi \otimes Q h^{2}$. Local asymptotic normality induces an inner product $\pi \otimes Q h h^{\prime}$ on $H$. Efficient estimators for a function $t(Q)$ are characterized in terms of the gradient of the functional with respect to this inner product. A functional $t(Q)$ of the transition distribution is differentiable, with gradient $g \in H$, if

$$
n^{1 / 2}\left\{t\left(Q_{n h}\right)-t(Q)\right\} \rightarrow \pi \otimes Q h g \quad \text { for } h \in H .
$$

The gradient is uniquely determined.

We can now characterize regular and efficient estimators of $t(Q)$. An estimator $T_{n}$ is called regular for $t(Q)$ in the full model, with limit $L$, if

$$
n^{1 / 2}\left\{T_{n}-t\left(Q_{n h}\right)\right\} \Rightarrow L \quad \text { under } P_{n h} \text { for } h \in H .
$$

A nonparametric version of Hájek's (1970) convolution theorem says that

$$
L=N_{g}+M,
$$

where $M$ is independent of $N_{g}$ and $N_{g}$ is normal with mean 0 and variance $\pi \otimes Q g^{2}$. This justifies calling $T_{n}$ efficient if $L=N_{g}$. It is a well-known consequence of the convolution theorem that an estimator $T_{n}$ is regular and efficient for $t(Q)$ if and only if

$$
n^{1 / 2}\left\{T_{n}-t(Q)\right\}=n^{-1 / 2} \sum_{i=1}^{n} g\left(X_{i-1}, X_{i}\right)+o_{P_{n}}(1) .
$$

We now characterize efficient estimators for a submodel of the full nonparameteric model above. Fix a transition distribution $Q(x, \mathrm{~d} y)$ which fulfils Assumptions 1 and 2. The local parameter space of the submodel is a subset $H_{0}$ of $H$ which we take to be closed and linear. A functional $t(Q)$ is differentiable on the submodel, with gradient $g \in H$, if (2.4) holds for $h \in H_{0}$. Now the gradient is not uniquely determined. A special role is played by the canonical gradient, the projection of an arbitrary gradient into $H_{0}$.

An estimator $T_{n}$ is called regular for $t(Q)$ in the submodel, with limit $L$, if (2.5) holds for $h \in H_{0}$. The convolution theorem then says that $L=N_{g_{0}}+M$, where $M$ and $N_{g_{0}}$ are independent. Hence $T_{n}$ is regular and efficient for $t(Q)$ in the submodel if and only if (2.6) holds with $g$ replaced by the canonical gradient $g_{0}$.

Call an estimator asymptotically linear for $t(Q)$ with influence function $k \in H$ if

$$
n^{1 / 2}\left\{T_{n}-t(Q)\right\}=n^{-1 / 2} \sum_{i=1}^{n} k\left(X_{i-1}, X_{i}\right)+o_{P_{n}}(1) .
$$

In these terms, an estimator is regular and efficient in the submodel if and only if it is asymptotically linear with influence function equal to the canonical gradient: 


$$
n^{1 / 2}\left\{T_{n}-t(Q)\right\}=n^{-1 / 2} \sum_{i=1}^{n} g_{0}\left(X_{i-1}, X_{i}\right)+o_{P_{n}}(1) .
$$

Moreover, an asymptotically linear estimator is regular if and only if its influence function is a gradient. A convenient reference for the convolution theorem and the characterizations of regular and efficient estimators is Greenwood and Wefelmeyer (1990).

\section{Restricting the invariant law}

In this section we consider a submodel described by a restriction on $\pi \otimes Q$. We have recalled in Section 2 that a regular and efficient estimator for the functional $t(Q)$ is characterized as an asymptotically linear estimator with influence function equal to the canonical gradient of $t(Q)$. We calculate the canonical gradient in two steps. First we describe the local parameter space $H_{0}$ in terms of the restriction on $\pi \otimes Q$. Then we calculate the projection of an arbitrary gradient into $H_{0}$.

Consider a submodel as in Section 2. Fix a transition distribution $Q(x, \mathrm{~d} y)$ in the submodel which fulfils Assumptions 1 and 2. Let $H_{0}$ denote a local parameter space at $Q$. Suppose that the submodel is described by a restriction on $\pi \otimes Q$. How can $H_{0}$ be described in terms of this restriction? To answer this, we perturb $\pi \otimes Q$ and interpret the restriction on the perturbation as a restriction on $H$. For smooth models it will be possible to choose, for each $h \in H_{0}$, a sequence $Q_{n h}$ in the submodel which is Hellinger differentiable in the sense of (2.1) and has the following two additional properties. Each $Q_{n h}$ has an invariant distribution $\pi_{n h}$, and $\pi_{n h} \otimes Q_{n h}$ admits a perturbation expansion. For $f \in L_{2}(\pi \otimes Q)$,

$$
n^{1 / 2}\left(\pi_{n h} \otimes Q_{n h} f-\pi \otimes Q f\right) \rightarrow \pi \otimes Q h \text { Af } \quad \text { for } h \in H_{0} .
$$

See Remark below. Here the operator $A$ is a kernel on $L_{2}(\pi \otimes Q)$ :

$$
A=I_{2}-\pi \otimes Q+(R-L) V,
$$

where $I_{2}$ is the identity on the space $L_{2}(\pi \otimes Q)$ of functions of two arguments, $R$ and $L$ map a function $v \in L_{2}(\pi)$ of one argument into functions of two arguments according to

$$
(R v)(x, y)=v(y), \quad(L v)(x, y)=v(x),
$$

and $V$ is the kernel given by

$$
V=\sum_{j=1}^{\infty}\left(Q^{j}-\pi \otimes Q\right)
$$

Writing

$$
(A f)(x, y)=f(x, y)-(Q f)(x)+\sum_{j=1}^{\infty}\left\{\left(Q^{j} f\right)(y)-\left(Q^{j+1} f\right)(x)\right\},
$$

we see that $A$ maps $L_{2}(\pi \otimes Q)$ into the local parameter space $H$. Let $L_{2,0}(\pi \otimes Q)$ denote the 
functions in $L_{2}(\pi \otimes Q)$ with expectation 0 under $\pi \otimes Q$. Since $A$ is the identity on $H$, it maps $L_{2,0}(\pi \otimes Q)$ onto $H$. Note that $V$ is similar to the potential $\sum_{j=0}^{\infty} Q^{J}$ and has the similar property:

$$
(I+V) Q=\pi \otimes Q+V
$$

Remark. In the full nonparametric model, $Q_{n h}$ has the additional properties described above if it is defined as in (2.2) and (2.3). Then

$$
\pi\left(Q_{n h} f-Q f\right)^{2}=n^{-1} \pi\left(Q h_{n} f\right)^{2} \leqslant 2 n^{-3 / 4} \pi f^{2} .
$$

Hence Assumption 2 holds and $Q_{n h}$ has an invariant distribution $\pi_{n h}$. Further, the perturbation expansion (3.1) follows from Lemma 2 of Greenwood and Wefelmeyer (1997), which is based on a version of a perturbation expansion of $\pi_{n h} f$ of Kartashov (1985a,b).

Let $B$ denote the adjoint of $A$. Then (3.1) reads

$$
n^{1 / 2}\left(\pi_{n h} \otimes Q_{n h} f-\pi \otimes Q f\right) \rightarrow \pi \otimes Q B h f \quad \text { for } h \in H_{0} .
$$

This means that the $\pi \otimes Q$ density of $\pi_{n h} \otimes Q_{n h}$ is approximately $1+n^{-1 / 2} B h$. Now we see how to translate a restriction on $\pi_{n h} \otimes Q_{n h}$ into a restriction on $h$ through a condition on $B h$. In the examples we have in mind, and in particular in Section 4,

$$
H_{0}=\{h \in H: B h \in F\}
$$

for some closed linear subspace $F$ of $L_{2}(\pi \otimes Q)$.

Once we have described the local parameter space $H_{0}$, we must calculate the canonical gradient of the functional $t(Q)$ that we want to estimate. According to Section 2, the canonical gradient $g_{0}$ is the projection of an arbitrary gradient $g \in H$ into $H_{0}$. It is therefore characterized by the properties that $g_{0} \in H_{0}$ and $g-g_{0}$ is orthogonal to $H_{0}$. The operator $A$ maps $L_{2,0}(\pi \otimes Q)$ onto $H$; so we can write $g=A f$ and $g_{0}=A f_{0}$ for some $f, f_{0} \in L_{2,0}(\pi \otimes Q)$. Hence the canonical gradient is characterized as the function $A f_{0}$ with $f_{0} \in L_{2,0}(\pi \otimes Q)$ such that $A f_{0} \in H_{0}$ and $A f-A f_{0}$ is orthogonal to $H_{0}$. The condition $A f_{0} \in H_{0}$ can, in turn, according to (3.5), be written as $B A f_{0} \in F$.

To describe the canonical gradient more usefully, it remains to calculate $B$ and $B A$. The adjoint $B$ will be written in terms of the reversed chain, with transition distribution $\bar{Q}(y, \mathrm{~d} x)$ defined by

$$
\pi(\mathrm{d} x) Q(x, \mathrm{~d} y)=\pi(\mathrm{d} y) \bar{Q}(y, \mathrm{~d} x)
$$

One checks that the transition distribution $\bar{Q}$ also has invariant distribution $\pi$.

For a function $f(x, y)$ of two arguments we shall follow the convention that the transition distribution of the reversed chain acts on $f$ from right to left, i.e. on the first argument of $f$ :

$$
(\bar{Q} f)(y)=\int \bar{Q}(y, \mathrm{~d} x) f(x, y)
$$


Similarly,

$$
\pi \otimes \bar{Q} f=\iint \pi(\mathrm{d} y) \bar{Q}(y, \mathrm{~d} x) f(x, y) .
$$

The kernel corresponding to $V$ for the reversed chain is

$$
\bar{V}=\sum_{j=1}^{\infty}\left(\bar{Q}^{j}-\pi \otimes \bar{Q}\right) .
$$

From (3.6) we obtain

$$
\pi(\mathrm{d} x) Q^{j}(x, \mathrm{~d} y)=\pi(\mathrm{d} y) \bar{Q}^{j}(y, \mathrm{~d} x) \quad \text { for } j=1,2, \ldots
$$

and therefore

$$
\pi(\mathrm{d} x) V(x, \mathrm{~d} y)=\pi(\mathrm{d} y) \bar{V}(y, \mathrm{~d} x) .
$$

The property corresponding to (3.3) is

$$
(I+\bar{V}) \bar{Q}=\pi \otimes \bar{Q}+\bar{V} .
$$

Let $B$ denote the kernel on $L_{2}(\pi \otimes Q)$,

$$
B=I_{2}-\pi \otimes Q+L(I+\bar{V})(\bar{Q}-Q) .
$$

By (3.9), on $H$ this kernel reduces to

$$
B=I_{2}+L(I+\bar{V}) \bar{Q}=I_{2}+L \bar{V} .
$$

Lemma 1. The adjoint of $A$ on $L_{2}(\pi \otimes Q)$ is $B$.

Lemma 2. We have $B A=I_{2}-\pi \otimes Q+R V+L \bar{V}$ on $L_{2}(\pi \otimes Q)$.

Lemmas 1 and 2 are proved in Section 5. We arrive at our description of the canonical gradient.

Theorem 1. Suppose that the local parameter space is of the form $H_{0}=\{h \in H: B h \in F\}$ for some closed linear subspace $F$ of $L_{2}(\pi \otimes Q)$. Let $t(Q)$ be differentiable, and let $g=$ Af be a gradient of $t(Q)$, with $f \in L_{2,0}(\pi \otimes Q)$.

Then the canonical gradient $g_{0}$ is characterized as the function of the form $g_{0}=A f_{0}$ with $f_{0} \in L_{2,0}(\pi \otimes Q)$ fulfilling

$$
\left(B A f_{0}=\right) f_{0}-\pi \otimes Q f_{0}+R V f_{0}+L \bar{V} f_{0} \in F
$$

and

$$
\pi \otimes Q A\left(f-f_{0}\right) h=0 \quad \text { for } h \in H_{0} .
$$

Example. An application of Theorem 1 is to the submodel of all Markov chains with invariant distribution in some parametric family $\pi_{\vartheta}, \vartheta \in \Theta$. Such a model arises when a 
diffusion is discretely observed and the diffusion model is parametric. For simplicitly, let $\Theta$ be an open subset of the real line. Fix a transition distribution $Q(x, \mathrm{~d} y)$ with invariant distribution $\pi_{\vartheta}$. Let $H_{0}$ denote the local parameter space at $Q$. The perturbation expansion (3.1) of $\pi_{n h} \otimes Q_{n h}$ implies a perturbation expansion of $\pi_{n h}$. For $f_{1} \in L_{2}\left(\pi_{\vartheta}\right)$,

$$
n^{1 / 2}\left(\pi_{n h} f_{1}-\pi_{\vartheta} f_{1}\right) \rightarrow \pi_{\vartheta} h A R f_{1}=\pi_{\vartheta} B h R f_{1} \quad \text { for } h \in H_{0} .
$$

On the other hand, $\pi_{n h}$ is in the parametric family and therefore approximately of the form $\pi_{\vartheta+n^{-1 / 2} u}$ for some $u$. Under a suitable smoothness assumption on the family, a Taylor expansion gives, for $f_{1} \in L_{2}\left(\pi_{\vartheta}\right)$,

$$
n^{1 / 2}\left(\pi_{\vartheta+n^{-1 / 2} u} f_{1}-\pi_{\vartheta} f_{1}\right) \rightarrow u \pi_{\vartheta} \ell_{\vartheta}^{\prime} f_{1}=u \pi_{\vartheta} \otimes Q R \ell_{\vartheta}^{\prime} R f_{1},
$$

where $\ell_{\vartheta}^{\prime}=\left.(\partial / \partial \tau)\left(\mathrm{d} \pi_{\tau} / \mathrm{d} \pi_{\vartheta}\right)\right|_{\tau=\vartheta}$. Hence

$$
H_{0}=\left\{h \in H: B h=u R \ell_{\vartheta}^{\prime} \text { for some } u\right\} .
$$

We can now apply Theorem 1 with $F=\left\{u R \ell_{y}^{\prime}: u \in \boldsymbol{R}\right\}$. For details see Kessler and Wefelmeyer (1997).

The following observations will be applied in Section 4. Since $Q$ and $\bar{Q}$ have invariant distribution $\pi$, so has

$$
Q^{S}=\frac{1}{2}(Q+\bar{Q})
$$

We have

$$
\pi(\mathrm{d} x) Q(x, \mathrm{~d} y)=\pi(\mathrm{d} y) \bar{Q}(y, \mathrm{~d} x)=\pi(\mathrm{d} x) \overline{\bar{Q}}(x, \mathrm{~d} y) .
$$

Hence $\overline{\bar{Q}}=Q$, and

$$
\begin{aligned}
\pi(\mathrm{d} x)\{Q(x, \mathrm{~d} y)+\bar{Q}(x, \mathrm{~d} y)\} & =\pi(\mathrm{d} y)\{\bar{Q}(y, \mathrm{~d} x)+\overline{\bar{Q}}(y, \mathrm{~d} x)\} \\
& =\pi(\mathrm{d} y)\{Q(y, \mathrm{~d} x)+\bar{Q}(y, \mathrm{~d} x)\}
\end{aligned}
$$

This means that $Q^{s}$ is reversible. The transition distribution $Q^{s}$ is called the additive reversibilization of $Q$ by Fill (1991) who uses reversibilizations to obtain bounds on the rate of convergence of Markov chains to stationarity.

\section{Reversible Markov chains}

Suppose that $X_{0}, \ldots, X_{n}$ come from a reversible Markov chain. This means that

$$
\pi(\mathrm{d} x) Q(x, \mathrm{~d} y)=\pi(\mathrm{d} y) Q(y, \mathrm{~d} x)
$$

and therefore $\bar{Q}=Q$, and $\pi \otimes Q$ is symmetric in the two components. We must keep in mind that $\bar{Q}$ still acts differently from $Q$ on functions of two arguments:

$$
(\bar{Q} f)(x)=\int \bar{Q}(x, \mathrm{~d} y) f(y, x)=(Q \bar{f})(x),
$$


where $\bar{f}(x, y)=f(y, x)$.

Fix a reversible transition distribution $Q(x, \mathrm{~d} y)$ which fulfils Assumptions 1 and 2. We have (3.4):

$$
n^{1 / 2}\left(\pi_{n h} \otimes Q_{n h} f-\pi \otimes Q f\right) \rightarrow \pi \otimes Q B h f \quad \text { for } h \in H .
$$

If $Q_{n h}$ as well as $Q$ are reversible, then $\pi_{n h} \otimes Q_{n h}$ and $\pi \otimes Q$ are symmetric. Therefore, $B h$ must also be symmetric. This suggests that the local parameter space for the model of reversible Markov chains is

$$
H_{0}=\{h \in H: B h \text { symmetric }\} .
$$

Indeed, if $h \in H_{0}$, then $\pi_{n h} \otimes Q_{n h}$ is symmetric up to $o\left(n^{-1 / 2}\right)$ by (4.2). It remains to check that for each $h \in H_{0}$ we can find a Hellinger differentiable sequence $Q_{n h}^{s}$ such that (4.2) holds and $\pi_{n h} \otimes Q_{n h}^{s}$ is exactly symmetric, so that $Q_{n h}^{s}$ is exactly reversible. This is the content of Lemma 3.

Lemma 3. For each $h \in H_{0}$ there exists a sequence of transition distributions $Q_{n h}^{s}$ with invariant distribution $\pi_{n h}$ such that $Q_{n h}^{s}$ is reversible (4.1) and Hellinger differentiable (2.1) with derivative $h$, and such that the perturbation expansion (3.1) holds for $\pi_{n h} \otimes Q_{n h}^{s}$.

The proof is in Section 5 .

Recall that the empirical estimator for $\pi \otimes Q f$ given by

$$
E_{n} f=\frac{1}{n} \sum_{i=1}^{n} f\left(X_{i-1}, X_{i}\right)
$$

is efficient in the full nonparametric model. To prove efficiency of the symmetrized empirical estimator $E_{n}^{s} f=E_{n} f_{s}$ with $f_{s}=\frac{1}{2}(f+\bar{f})$ and $\bar{f}(x, y)=f(y, x)$ in the model given by all reversible Markov chains, we apply Theorem 1.

Theorem 2. For $f \in L_{2}(\pi \otimes Q)$, the estimator

$$
E_{n} f_{s}=\frac{1}{2 n} \sum_{i=1}^{n}\left\{f\left(X_{i-1}, X_{i}\right)+f\left(X_{i}, X_{i-1}\right)\right\}
$$

is regular and efficient for $\pi \otimes Q f$ in the model given by all reversible Markov chains.

Proof. By Lemma 3 we have $H_{0}=\{h \in H: B h$ symmetric $\}$. Hence the assumption on $H_{0}$ of Theorem 1 holds with

$$
F=\left\{f \in L_{2}(\pi \otimes Q): f \text { symmetric }\right\} .
$$

We want to show that $A f_{s}$ is the canonical gradient of the functional $t(Q)=\pi \otimes Q f$. By the perturbation expansion (3.1), $g=A f$ is a gradient of $\pi \otimes Q f$. It suffices to check that the two conditions on $f_{0}$ in Theorem 1 hold for $f_{0}=f_{s}$. For the first condition, we observe that $R V f_{s}+L \bar{V} f_{s}$ is symmetric since $f_{s}$ is symmetric. The second condition holds since by Lemma 1 we have, for all $h \in H_{0}$, 


$$
\pi \otimes Q\left(A f-A f_{s}\right) h=\frac{1}{2} \pi \otimes Q A(f-\bar{f}) h=\frac{1}{2} \pi \otimes Q(f-\bar{f}) B h=0 .
$$

The last equality holds because $B h$ is symmetric for $h \in H_{0}$ and $\pi \otimes Q$ is symmetric, and therefore

$$
\pi \otimes Q f B h=\pi \otimes Q \bar{f} B h .
$$

Hence $A f_{s}$ is the canonical gradient, and the characterization (2.8) says that an estimator for $\pi \otimes Q f$ is regular and efficient if it is asymptotically linear with influence function $A f_{s}$.

We have

$$
n^{1 / 2}\left(E_{n} f_{s}-\pi \otimes Q f\right)=n^{-1 / 2} \sum_{i=1}^{n}\left\{f_{s}\left(X_{i-1}, X_{i}\right)-\pi \otimes Q f\right\} .
$$

By the martingale approximation of Gordin and Lifšic (1978) we have, for $f \in L_{2,0}(\pi \otimes Q)$,

$$
n^{-1 / 2} \sum_{i=1}^{n} f\left(X_{i-1}, X_{i}\right)=n^{-1 / 2} \sum_{i=1}^{n}(A f)\left(X_{i-1}, X_{i}\right)+o_{P_{n}}(1) .
$$

This approximation, applied to $f=f_{s}-\pi \otimes Q f$, shows that $E_{n} f_{s}$ has the influence function $A f_{s}$.

\section{Proofs of the lemmas}

Proof of Lemma 1. We must prove that, for $e, f \in L_{2}(\pi \otimes Q)$,

$$
\pi \otimes Q e \text { Af }=\pi \otimes Q B e f .
$$

Clearly, $I_{2}$ is its own adjoint. We prove that $L(\bar{Q}-Q)$ is the adjoint of $(R-L)(Q-\pi \otimes Q)$ and $L\left(\bar{Q}^{j-1}-\pi \otimes \bar{Q}\right)(\bar{Q}-Q)$ is the adjoint of $(R-L)\left(Q^{j}-\pi \otimes Q\right)$ for $j=2,3, \ldots$ We can omit the centring by $\pi \otimes Q$ because $(R-L) \pi \otimes Q=0$. We can omit the centring by $\pi \otimes \bar{Q}$ because also

$$
\begin{aligned}
\pi \otimes \bar{Q}(\bar{Q}-Q) e & =\int \pi(\mathrm{d} x) \bar{Q}(x, \mathrm{~d} y)\left(\int \bar{Q}(y, \mathrm{~d} z) e(y, z)-\int Q(y, \mathrm{~d} z) e(y, z)\right) \\
& =\int \pi(\mathrm{d} y)\left(\int Q(y, \mathrm{~d} z) e(y, z)-\int Q(y, \mathrm{~d} z) e(y, z)\right) \\
& =0 .
\end{aligned}
$$

Now

$$
\begin{aligned}
\pi \otimes Q e(R-L) Q f & =\iint \pi(\mathrm{d} x) Q(x, \mathrm{~d} y) e(x, y)\left(\int Q(y, \mathrm{~d} z) f(y, z)-\int Q(x, \mathrm{~d} z) f(x, z)\right) \\
& =\int \pi(\mathrm{d} y) \int \bar{Q}(y, \mathrm{~d} x) e(x, y) \int Q(y, \mathrm{~d} x) f(y, z)
\end{aligned}
$$




$$
\begin{aligned}
& -\int \pi(\mathrm{d} x) \int Q(x, \mathrm{~d} y) e(x, y) \int Q(x, \mathrm{~d} z) f(x, z) \\
= & \iint \pi(\mathrm{d} x) Q(x, \mathrm{~d} y)(\bar{Q} e)(x) f(x, y) \\
& -\iint \pi(\mathrm{d} x) Q(x, \mathrm{~d} y)(Q e)(x) f(x, y) \\
= & \pi \otimes Q L(\bar{Q}-Q) e f
\end{aligned}
$$

and, for $j=2,3, \ldots$,

$$
\begin{aligned}
\pi \otimes Q e(R-L) Q^{j} f= & \iint \pi(\mathrm{d} x) Q(x, \mathrm{~d} y) e(x, y)\left(\int Q^{j-1}(y, \mathrm{~d} z) \int Q(z, \mathrm{~d} w) f(z, w)\right. \\
& \left.-\int Q^{j-1}(x, \mathrm{~d} z) \int Q(z, \mathrm{~d} w) f(z, w)\right) \\
= & \int \pi(\mathrm{d} y) \int \bar{Q}(y, \mathrm{~d} x) e(x, y) \int Q^{j-1}(y, \mathrm{~d} z) \int Q(z, \mathrm{~d} w) f(z, w) \\
& -\int \pi(\mathrm{d} z) \int \bar{Q}^{j-1}(z, \mathrm{~d} x) \int Q(x, \mathrm{~d} y) e(x, y) \int Q(z, \mathrm{~d} w) f(z, w) \\
= & \int \pi(\mathrm{d} z) \int \bar{Q}^{j-1}(z, \mathrm{~d} y) \int \bar{Q}(y, \mathrm{~d} x) e(x, y) \int Q(z, \mathrm{~d} w) f(z, w) \\
& -\int \pi(\mathrm{d} z) \int \bar{Q}^{j-1}(z, \mathrm{~d} x) \int Q(x, \mathrm{~d} y) e(x, y) \int Q(z, \mathrm{~d} w) f(z, w) \\
= & \pi \otimes Q L \bar{Q}^{j-1}(\bar{Q}-Q) e f .
\end{aligned}
$$

Proof of Lemma 2. Note that $Q R=Q$ and $Q L=I_{1}$ on $L_{2}(\pi)$, where $I_{1}$ is the identity on $L_{2}(\pi)$. Hence $Q^{j} R=Q^{j}$ and $Q^{j} L=Q^{j-1}$ on $L_{2}(\pi)$ for $j=2,3, \ldots$, and therefore

$$
V L=\sum_{j=1}^{\infty}\left(Q^{j} L-\pi\right)=I_{1}-\pi+V R .
$$

For $\bar{V}$ we have the corresponding relation

$$
\bar{V} R=I_{1}-\pi+\bar{V} L .
$$

Hence

$$
\bar{V}(R-L)=I_{1}-\pi,
$$


so that

$$
\bar{V} A=\bar{V}+\bar{V}(R-L) V=\bar{V}+V
$$

and, with (3.10),

$$
\begin{aligned}
B A & =\left(I_{2}+L \bar{V}\right) A \\
& =I_{2}-\pi \otimes Q+(R-L) V+L \bar{V}+L V \\
& =I_{2}-\pi \otimes Q+R V+L \bar{V} .
\end{aligned}
$$

Proof of Lemma 3. Let $Q_{n h}$ be the sequence $Q(x, \mathrm{~d} y)\left\{1+n^{-1 / 2} h_{n}(x, y)\right\}$ defined in (2.2) and (2.3). By the Remark in Section 3, each transition distribution $Q_{n h}$ has an invariant distribution $\pi_{n h}$, and $\pi_{n h} \otimes Q_{n h}$ admits the perturbation expansion (3.1). The transition distribution $\bar{Q}_{n h}$ of the reversed chain is defined by (3.6):

$$
\pi_{n h}(\mathrm{~d} x) Q_{n h}(x, \mathrm{~d} y)=\pi_{n h}(\mathrm{~d} y) \bar{Q}_{n h}(y, \mathrm{~d} x) .
$$

Let $Q_{n h}^{s}=\frac{1}{2}\left(Q_{n h}+\bar{Q}_{n h}\right)$ be the additive reversibilization of $Q_{n h}$. We have seen at the end of Section 3 that $Q_{n h}^{s}$ has invariant distribution $\pi_{n h}$ and is reversible.

By (5.1), (3.1), Lemma 1, reversibility of $Q$ and symmetry of $B h$,

$$
\begin{aligned}
\iint \pi_{n h}(\mathrm{~d} x) \bar{Q}_{n h}(x, \mathrm{~d} y) f(x, y) & =\pi_{n h} \otimes Q_{n h} \bar{f} \\
& =\pi \otimes Q \bar{f}+n^{-1 / 2} \pi \otimes Q h A \bar{f}+o\left(n^{-1 / 2}\right) \\
& =\pi \otimes Q f+n^{-1 / 2} \pi \otimes Q B h f+o\left(n^{-1 / 2}\right) \\
& =\pi \otimes Q f+n^{-1 / 2} \pi \otimes Q h A f+o\left(n^{-1 / 2}\right) .
\end{aligned}
$$

Combining with (3.1), we obtain the perturbation expansion

$$
n^{1 / 2}\left(\pi_{n h} \otimes Q_{n h}^{s} f-\pi \otimes Q f\right) \rightarrow \pi \otimes Q h \text { Af. }
$$

To prove that $Q_{n h}^{s}$ is Hellinger differentiable, we must show that $\pi_{n h}$ has a $\pi$ density. On $L_{2}(\pi)$ we have

$$
\pi_{n h}=\pi\left\{I-\left(Q_{n h}-Q\right) D\right\}^{-1}
$$

with

$$
D=I+\sum_{j=1}^{\infty}\left(Q^{j}-\pi\right)
$$

(see Greenwood and Wefelmeyer (1997, Lemma 2)). Write $Q_{n h}-Q=n^{-1 / 2} Q_{h_{n}}$ with $Q_{h}(x, \mathrm{~d} y)=Q(x, \mathrm{~d} y) h(x, y)$. Then

$$
\pi_{n h} f=\pi f+\sum_{k=1}^{\infty} n^{-k / 2} \pi\left(Q_{h_{n}} D\right)^{k} f .
$$


As in the proof of Lemma 1 we can show that

$$
\pi Q_{h_{n}} D f=\pi \otimes Q h_{n} R D f=\pi \bar{V} h_{n} f .
$$

By induction,

$$
\pi\left(Q_{h_{n}} D\right)^{k} f=\pi W^{k} h_{n} f \quad \text { for } k=1,2, \ldots
$$

with

$$
W h=\bar{V} h, \quad W^{k} h=\bar{V}\left(L W^{k-1} h h\right) \text { for } k=2,3, \ldots
$$

By Assumption 2, the operator $\bar{V}$ is bounded on $L_{2}(\pi)$. We obtain a representation for the $\pi$ density of $\pi_{n h}$,

$$
\begin{aligned}
\frac{\mathrm{d} \pi_{n h}}{\mathrm{~d} \pi} & =1+\sum_{k=1}^{\infty} n^{-k / 2} W^{k} h_{n} \\
& =1+n^{-1 / 2}\left(\bar{V} h_{n}+s_{n}\right)
\end{aligned}
$$

with

$$
s_{n}=\sum_{k=2}^{\infty} n^{-(k-1) / 2} W^{k} h_{n} .
$$

It is straightforward to check that $\pi s_{n}^{2} \rightarrow 0$ and that $\bar{V} h_{n}$ and $s_{n}$ are bounded by $O\left(n^{1 / 8}\right)$.

From (5.1) and (5.2)

$$
\begin{aligned}
\pi_{n h}(\mathrm{~d} x) Q_{n h}(x, \mathrm{~d} y) & =\pi_{n h}(\mathrm{~d} y) \bar{Q}_{n h}(y, \mathrm{~d} x) \\
& =\pi(\mathrm{d} y)\left[1+n^{-1 / 2}\left\{\left(\bar{V} h_{n}\right)(y)+s_{n}(y)\right\}\right] \bar{Q}_{n h}(y, \mathrm{~d} x) .
\end{aligned}
$$

On the other hand, by definition (2.2) of $Q_{n h}$,

$$
\pi_{n h}(\mathrm{~d} x) Q_{n h}(x, \mathrm{~d} y)=\pi(\mathrm{d} x)\left[1+n^{-1 / 2}\left\{\left(\bar{V} h_{n}\right)(x)+s_{n}(x)\right\}\right] Q(x, \mathrm{~d} y)\left\{1+n^{-1 / 2} h_{n}(x, y)\right\} .
$$

Use $\pi(\mathrm{d} x) Q(x, \mathrm{~d} y)=\pi(\mathrm{d} y) Q(y, \mathrm{~d} x)$ and solve for $\bar{Q}_{n h}$ to obtain that

$$
\begin{aligned}
\bar{Q}_{n h}(y, \mathrm{~d} x)= & Q(y, \mathrm{~d} x)\left\{1+n^{-1 / 2} h_{n}(x, y)\right\} \\
& \times\left[1+n^{-1 / 2}\left\{\left(\bar{V} h_{n}\right)(x)+s_{n}(x)\right\}\right] \\
& \times\left[1+n^{-1 / 2}\left\{\left(\bar{V} h_{n}\right)(y)+s_{n}(y)\right\}\right]^{-1} .
\end{aligned}
$$

Hence $\bar{Q}_{n h}$ is Hellinger differentiable (2.1) with derivative $\bar{h}+R \bar{V} h-L \bar{V} h$. We note that relation (3.10) implies that

$$
B h=h+L \bar{V} h=\bar{h}+R \bar{V} h \quad \text { for } h \in H_{0} .
$$

Therefore, the derivative equals $h$. Hence $Q_{n h}^{s}$ is also Hellinger differentiable with derivative $h$.

Remark. The arguments in the proof of Lemma 3 could be used to obtain a general stability 
result for Markov chains of the following type. If $Q^{\prime}$ has a $Q$ density and is close to $Q$, then the invariant distribution $\pi^{\prime}$ of $Q^{\prime}$ has a $\pi$ density, and the density of $\pi^{\prime}$ has a representation in terms of the density of $Q^{\prime}$.

\section{Acknowledgements}

We thank the referees and an Associate Editor for suggestions which led to substantial improvements of the presentation. This work was supported by the Natural Sciences and Engineering Research Council of Canada.

\section{References}

Aït-Sahalia, Y. (1996) Nonparametric pricing of interest rate derivative securities. Econometrica, 64, $527-560$.

Bibby, B.M. and Sørensen, M. (1995) Martingale estimating functions for discretely observed diffusion processes. Bernoulli, 1, 17-39.

Bickel, P.J. (1993) Estimation in semiparametric models. In C.R. Rao (ed.), Multivariate Analysis: Future Directions, pp. 55-73. Amsterdam: North-Holland.

Drost, F.C. and Klaassen, C.A.J. (1996) Efficient estimation in semiparametric GARCH models. J. Econometrics., 81, 193-221.

Fill, J.A. (1991) Eigenvalue bounds on convergence to stationarity for nonreversible Markov chains, with an application to the exclusion process. Ann. Appl. Probab., 1, 62-87.

Gordin, M.I. and Lifšic, B.A. (1978) The central limit theorem for stationary Markov processes. Sov. Math. Dokl., 19, 392-394.

Greenwood, P.E. and Wefelmeyer, W. (1990) Efficiency of estimators for partially specified filtered models. Stochastic Processes Applic., 36, 353-370.

Greenwood, P.E. and Wefelmeyer, W. (1995) Efficiency of empirical estimators for Markov chains. Ann. Statist., 23, 132-143.

Greenwood, P.E. and Wefelmeyer, W. (1997) Maximum likelihood estimator and Kullback-Leibler information in misspecified Markov chain models. Teor. Veroyatnost. Primenen, 42, 169-178.

Hájek, J. (1970) A characterization of limiting distributions of regular estimates. Z. Wahrscheinlichkeitstheorie Verw. Geb., 14, 323-330.

Hansen, L.P. and Scheinkman, J.A. (1995) Back to the future: generating moment implications for continuous-time Markov processes. Econometrica, 63, 767-804.

Höpfner, R. (1993) On statistics of Markov step processes: representation of log-likelihood ratio processes in filtered local models. Probab. Theory Related Fields, 94, 375-398.

Höpfner, R., Jacod, J. and Ladelli, L. (1990) Local asymptotic normality and mixed normality for Markov statistical models. Probab. Theory Related Fields, 86, 105-129.

Jeganathan, P. (1995) Some aspects of asymptotic theory with applications to time series models. Econometric Theory, 11, 818-887.

Kartashov, N.V. (1985a) Criteria for uniform ergodicity and strong stability of Markov chains with a common phase space. Theory Probab. Math. Statist., 30, 71-89.

Kartashov, N.V. (1985b) Inequalities in theorems of ergodicity and stability for Markov chains with common phase space. I. Theory Probab. Applic., 30, 247-259. 
Kessler, M., Schick, A. and Wefelmeyer, W. (1997) The information in the marginal law of a Markov chain. Preprint.

Koul, H.L. and Schick, A. (1997) Efficient estimation in nonlinear autoregressive time series models. Bernoulli, 3, 247-277.

Kreiss, J.-P. (1987) On adaptive estimation in stationary ARMA processes. Ann. Statist., 15, 112-133.

Pedersen, A.R. (1995) Consistency and asymptotic normality of an approximate maximum likelihood estimator for discretely observed diffusion processes. Bernoulli, 1, 257-279.

Penev, S. (1991) Efficient estimation of the stationary distribution for exponentially ergodic Markov chains. J. Statist. Plann. Inference, 27, 105-123.

Wefelmeyer, W. (1996) Quasi-likelihood models and optimal inference. Ann. Statist., 24, 405-422.

Received August 1996 and revised June 1997 